Recherches en didactique des langues et des cultures

Les cahiers de l'Acedle

$4 \mid 2007$

Notions en questions : Méthodologies de recherche

\title{
Du positionnement épistémologique aux données de terrain
}

Françoise Demaizière et Jean-Paul Narcy-Combes

\section{OpenEdition}

Édition électronique

URL : https://journals.openedition.org/rdlc/4850

DOI : $10.4000 /$ rdlc. 4850

ISSN : $1958-5772$

Éditeur

ACEDLE

Référence électronique

Françoise Demaizière et Jean-Paul Narcy-Combes, « Du positionnement épistémologique aux données de terrain », Recherches en didactique des langues et des cultures [En ligne], 4 | 2007, mis en ligne le 21 juin 2007, consulté le 28 juin 2022. URL : http://journals.openedition.org/rdlc/4850 ; DOI https://doi.org/10.4000/rdlc. 4850

Ce document a été généré automatiquement le 29 septembre 2020.

\section{$\Theta \Theta \Theta$}

Recherches en didactique des langues et des cultures is licensed under a Creative Commons AttributionNonCommercial-NoDerivatives 4.0 International License 


\title{
Du positionnement épistémologique aux données de terrain
}

\author{
Françoise Demaizière et Jean-Paul Narcy-Combes
}

\section{Introduction - Méthodologie et méthode}

1 Le concept examiné pour cette rencontre est la méthodologie de recherche en didactique des langues. Pourquoi ne pas s'être arrêté sur la notion de méthode ? Voilà sans doute la première question à laquelle il convient de répondre.

2 Une méthode s'impose à un exécutant qui doit la suivre, c'est bien ce que Descartes (1636) affirmait. Pour lui, il convient de reconstruire les sciences selon un plan qui les unifie et les installe sur des fondements communs, grâce à l'unité d'une méthode. Il est d'abord nécessaire de faire table rase des opinions ou préjugés qui encombrent l'esprit et viennent, dans son cas, d'une éducation éclectique, ensuite de définir rigoureusement la méthode que l'on compte appliquer dans les sciences et dont les règles sont au nombre de quatre, l'évidence, l'ordre, l'analyse, l'énumération. L'aboutissement de cette vision a été, au dix-neuvième siècle, le positivisme qui en est arrivé à un positionnement où l'on sait d'emblée ce qu'il convient de faire.

3 C'est ce que Depover, Giardina et Marton (1998: 36) nous confirment:"La méthode, c'est l'ensemble des démarches, des cheminements ordonnés et rationnels, fixant le mode d'intervention." De même, Puren (1988: 17) : "[une] méthode [est] un ensemble de procédés et de techniques (...) visant à susciter (...) un comportement ou une activité déterminée".

4 Par contre, parler de méthodologie oriente vers une pratique réflexive, menée en fonction de critères reconnus par une communauté (de chercheurs) mais sans que l'on postule d'unicité des réponses possibles et donc du paradigme de référence. La méthodologie permet, à partir d'un corps de principes ou de repères reconnus, de construire une action (de recherche pour nous ici) adaptée au contexte spécifique dans lequel elle se met en place, alors que la méthode semble présupposer un paradigme unique. C'est ce que suggère déjà Puren (1988: 17) quand il propose de parler de méthodologie d'enseignement des langues pour 
un ensemble cohérent de procédés, techniques et méthodes qui s'est révélé capable, sur une certaine période historique et chez des concepteurs différents de générer des cours relativement originaux par rapport aux cours antérieurs.

5 Pour nous, la recherche en sciences humaines, et donc en didactique, ne peut s'appuyer sur une méthode. Il lui faut se référer à des méthodologies permettant de construire un protocole pertinent en fonction des divers paramètres en jeu. Même s'il est fréquent en France, ce point de vue est loin d'être général, et dans ce que les anglophones appellent Applied linguistics de nombreux débats sur la validité d'une méthode ou d'une méthodologie ont marqué les trente dernières années. Seidlhofer (2003) en a regroupés quelques-uns dans un ouvrage au titre parlant de Controversies in Applied Linguistics et l'ouvrage de Jordan (2004), Theory Construction in Second Language Acquisition, montre que le débat est loin d'être clos. L'analyse de ce que Seidlhofer appelle Controversy 7 montre que ce débat est particulièrement vif entre des chercheurs tels que Long, d'une part et Firth et Wagner d'autre part (Seidlhofer 2003 : 169-269). Nous l'évoquons en raison de cette vivacité, du renom de ces chercheurs dans le monde anglophone et parce que ce débat est public. Le CNRS reproduit des phénomènes semblables (Mucchielli, 2000), ce n'est pas sans incidence sur les possibilités de publication et le déroulement des carrières, et donc sur les motivations.

6 Quelles en sont les causes et les conséquences, qu'est ce qui oppose les chercheurs qui se reconnaissent en Long et ceux qui comprennent mieux Firth et Wagner?

7 Nous positionnant clairement du côté de la méthodologie plutôt que des méthodes, nous allons suggérer que la réponse pourrait se situer dans la tension entre le positionnement épistémologique du chercheur, les données qu'il considère, et la façon dont il les traite. Nous insisterons sur le positionnement épistémologique nécessaire à tout chercheur, ce positionnement est ou non explicité mais il est toujours sous-jacent. Il influence le recueil et le traitement des données et la méthodologie de recherche.

Nous nous demanderons d'abord ce que sont la didactique et la didactique des langues dans leur positionnement épistémologique (paragraphe 2).

\section{Positionnement épistémologique et didactique}

\subsection{Définition du positionnement épistémologique}

9 Nous avons parlé de positionnement épistémologique dans le point précédent sans définir ce que nous entendions par ces mots!

10 Le mot "épistémologie" est bien entendu polysémique, il englobe les méthodes propres à chaque science ou domaine scientifique, tout comme les démarches de la pensée scientifique en général, mais il peut se référer également au problème de la vérité scientifique. Il est tentant de garder tous ces sens, dans une acception synthétique, qui rappellerait que l'épistémologie est la réflexion sur la construction et la gestion du savoir dans un domaine donné et dans son rapport avec les autres domaines de la réflexion scientifique. Nous serons encore plus larges en considérant que l'épistémologie s'intéresse aussi à la façon dont la connaissance se construit pour chaque individu; c'est alors que le mot "positionnement" prend son sens.

11 G. Bachelard (1936) a montré la nécessité d'une rupture épistémologique pour passer d'une explication "toute prête" d'un phénomène, suscitée par divers conditionnements 
ou habitudes, à une compréhension qui s'appuie sur une théorie ou une approche scientifiques. Ce qui gêne ce passage, c'est l'obstacle épistémologique, qui est conçu comme une entrave à la connaissance scientifique, entrave inhérente à la construction du savoir lui-même, et donc aux représentations naïves que l'on se fait initialement, et qui ne vient pas de difficultés liées à l'objet. Par exemple, il n'est pas difficile de comprendre que la terre est sphérique, mais il a été difficile de l'accepter, pendant des siècles, car elle ne semble pas l'être et que cela remettait des croyances en cause.

12 Il importe donc de cerner, dans chaque domaine, ce qui peut faire obstacle à une approche scientifique, et de voir comment les façons de gérer ces obstacles expliquent les positionnements épistémologiques des uns et des autres.

\subsection{Définition de la didactique}

Nous partirons de la définition de Bailly (1997: 10), qui nous a toujours paru adéquate.

Le terme "Didactique", dans son acception moderne - relativement récente renvoie, au moins, à une démarche de distanciation et, au plus, à une pleine activité de théorisation : schématiquement, il s'agit dans tous les cas, pour un observateur ou un expérimentateur, de s'abstraire de l'immédiateté pédagogique et d'analyser à travers toutes ses composantes l'objet d'enseignement, les buts poursuivis dans l'acte pédagogique, les stratégies utilisées par l'enseignement, les transformations de compétences et de conduites que cet enseignement induit chez l'élève et par conséquent les stratégies d'appropriation de l'objet déployées par cet élève lors de son activité d'apprentissage

Cette définition nous conduit vers une série de réflexions. Puisqu'il s'agit autant d'observer que d'agir, on peut admettre que l'objet de la didactique relève aussi bien de la description que de l'action, tout en restant dans le champ de la praxéologie. Précisons également que nous nous arrêtons aux apprentissages en milieu institutionnel, là est la limite de la didactique pour nous.

\subsection{Didactique, distanciation, objectivité et intérêts du chercheur}

a référence à la distanciation, qui est la première étape que donne Bailly, ne peut que nous orienter vers Elias (Engagement et distanciation, 1993). Cette distanciation implique-telle un simple recul ou exige-t-elle le repérage de ce qui détermine nos valeurs, nos comportements mais également notre respect d'une méthodologie? En période de "science normale", c'est-à-dire en période d'accord sur le paradigme dominant, au sens de Kuhn (1970), ce respect est facile à suivre, mais il nous faudra déterminer s'il en va de même en didactique.

En nous permettant de paraphraser Lacroix (2002), rappelons qu'Engagement et distanciation est un modèle de rationalisation et de systématisation de l'effort de retour sur soi. La "distanciation" touche à la question de la "rationalité" et à la question de "l'objectivité". Cette dernière n'est pas une simple exigence de neutralité dans l'action et le jugement, elle ne s'oppose pas à la subjectivité de la langue commune. Elle est liée à la question des "valeurs" du didacticien (dans notre cas). Elle n'est pas une difficulté ou un obstacle dont il s'agirait de prévenir les effets mais une donnée du problème posé, qui doit être prise en compte, quitte à ne pas en rester à cette observation préliminaire. C'est donc le travail scientifique dans sa totalité qui se trouve pratiquement bouleversé - en même temps qu'il se trouve redéfini - à l'instant où son objet ne peut plus être posé en 
stricte extériorité (ce qui ne veut pas dire qu'il n'ait pas une forme d'objectivité). Ce retour sur soi, appelé par ce que la tradition nomme, à tort, l'examen de l'objet, en obligeant à inclure dans la réflexion l'investissement que le scientifique met dans l'objet de sa curiosité (qui relève de sa subjectivité) peut conduire notamment à une forme de défiance. Pourtant, pour le chercheur, bien de ses efforts intellectuels, même les mieux intentionnés, sont enclins - sans qu'il se l'avoue - à épouser beaucoup plus qu'à éprouver les préjugés de tous ceux dont il souhaite se faire connaître et reconnaître.

Rappelons Callon et Latour (1991) dont les propos sur la conduite de la science, d'une façon plus polémique parfois, rejoignent ceux d'Elias quand ce dernier postule que la recherche résulterait d'une conjonction de la curiosité (intellectuelle) du chercheur et de ses intérêts (sociaux).

En acquisition des langues, Jordan (2004: 83) écrit que l'objectivité de la connaissance scientifique est issue de sa nature de construit social créé par une communauté. Il affirme que la pratique de la science est consensuelle dans une rationalité partagée (2004: 88). Nous comprenons que, de son point de vue, l'objectivité relève bien du respect du fonctionnement de la communauté, ce qui rejoint l'analyse de Kuhn (1970) sur les périodes de science normale, mais est-ce le cas pour la didactique ? Par contre, l'analyse que fait Jordan de la subjectivité ne lie pas celle-ci à l'engagement, issu de la combinaison de la curiosité et des "intérêts" du chercheur et de ce qui les explique et en cela il diffère d'Elias et nous paraît moins convaincant sur ce point. Elias nous conduirait presque à écrire que l'explicitation de ce qui est subjectif, et la mesure des effets de la subjectivité assurent l'objectivité si l'on respecte une méthodologie attestée.

Pour reprendre ce point, la méthodologie est ce qui assure la distanciation du chercheur et son objectivité, mais en didactique, comme dans les autres domaines des sciences humaines, le consensus dont parle Jordan n'est pas en place, ce qui complexifie le travail du chercheur dans sa quête de reconnaissance.

\subsection{Didactique et théorisation}

Bailly parle dans sa définition de "pleine théorisation". Cet objectif paraît souvent ambitieux en didactique. La citation ci-dessous de Soulet (2003) à propos des sciences sociales s'applique largement.

Toute théorisation de la pratique du travail social est illusoire parce que, en cherchant à conceptualiser les raisons d'agir, la discussion théorique s'éloigne fortement de cette pratique ou parce que, en cherchant à modéliser les formes de l'agir, la description abstraite ne parvient pas au statut d'explication théorique, limitant son apport à une catégorisation formelle. Il convient donc de renoncer à l'ambition d'une théorisation spécifique. Cette dé-singularisation ne signifie pas de renoncer à produire une explication théorique de ce qu'est le fait d'agir au sein $d u$ travail social, mais vise à construire une théorie de l'action tenant compte des caractéristiques formelles de ce contexte particulier dans lequel cette action se déroule.

Soulet soulève ici une question importante: peut-il y avoir une théorisation décontextualisée?

Si tout est contextualisé, alors ne seraient possibles que des recherches de nature idiographique (on ne peut universaliser), et on ne serait pas en mesure d'aboutir à des théories universalisantes (nomothétie). Or, en sciences humaines et sociales, coexistent des approches qui se veulent générales et explicatives (cf. Durkheim, 1894), alors que, au 
contraire, d'autres chercheurs se situent dans la compréhension et l'étude des individus dans leur spécificité (cf. Weber, 1905), pour ne pas parler de ceux qui se focalisent sur l'interaction. Ces points de vue sont-ils antagonistes et exclusifs, ou des dialogues sont-ils envisageables? de recrutement et les pratiques institutionnelles, il y a eu mise en place d'enseignements de didactique. Or, au niveau de la professionnalisation, il semble que l'appui sur des références n'est pas nécessairement soumis à questionnement scientifique. Les instructions "officielles", qui conditionnent l'enseignement des langues dans les établissements scolaires français, proposent plutôt des techniques rationalisées qu'une authentique réflexion scientifique ou une authentique méthodologie permettant au praticien des prises de recul et d'initiative à partir de références issues de la recherche. On ne s'en étonnera pas outre mesure. N'est-ce pas à la communauté des chercheurs en didactique de montrer l'opportunité d'une réflexion scientifique rigoureuse?

\subsection{Constituer et situer la didactique}

l'on regarde l'évolution du domaine, on voit qu'en parallèle à l'approche dite "communicative", sont apparus plusieurs courants, l'un vient de la linguistique, principalement énonciativiste (cf. Bailly, 1997) ${ }^{1}$ un autre courant est historique (cf. Puren, 1994), un troisième est centré sur l'analyse des pratiques, en lien avec les recherches en acquisition des langues. Il y a également, de plus en plus perceptibles, avec les travaux du Conseil de l'Europe et l'"approche actionnelle", entre autres, une didactique qui s'appuie sur le plurilinguisme.

Face à cette diversité, il reste à ce jour difficile de constituer une didactique des langues, ainsi certaines thèses du domaine témoignent parfois d'une certaine difficulté à se situer. Une compréhension rigoureuse des processus d'"intervention pédagogique", terme qu'il conviendra de redéfinir, et d'apprentissage, appréhendés dans leur dialectique, peut permettre à la didactique de jouer un rôle de discipline scientifiquement étayée. Sinon, elle proposera des techniques, à partir desquelles on peut mettre en place ce que l'on appelait des méthodes. Si les positions institutionnelles et les pratiques ne sont pas 
toujours soumises à un questionnement scientifique, c'est qu'il n'y a pas d'authentique rupture épistémologique. Il se pourrait que les obstacles proviennent de l'apparente scientificité des termes employés : langue, production, apprentissage, acquisition, action, pour ne citer que ceux-là.

Tant en ce qui concerne les pratiques que la recherche, on se trouve face à un foisonnement de théories, et donc de définitions des termes ci-dessus en particulier (et ils ne sont pas les seuls). Rien qu'au niveau de l'acquisition des langues, Jordan cite plus de 60 théories (2004:360). Face à l'absence de paradigme dominant, chaque chercheur est amené à trouver sa méthodologie, à se trouver une méthodologie, mais également à définir ses termes. Il a un contexte à prendre en compte et nous avons vu qu'il aura un positionnement à déterminer, ce qui l'orientera vers des choix méthodologiques.

Nous remarquerons que dans ce qu'on appelle DNL (disciplines non linguistiques), on s'intéresse plus à la didactique de la discipline enseignée et à l'acquisition seule. Dans un master 2 recherche de didactique des langues, il y a authentiquement disciplinarisation de la didactique des langues: c'est bien elle qu'on enseigne. Cela modifie-t-il la perspective ? On peut au moins souhaiter que cela conduise à expliciter les références et à sensibiliser les étudiants à opérer la rupture épistémologique que nous soulignons ici.

Le paradoxe est que, même si on ne pose pas la question en termes institutionnels, on peut légitimement s'interroger sur la nature des liens de la didactique avec les sciences du langage, les sciences de l'éducation et les autres domaines de référence (cf. 3.1. cidessous). On ne peut employer de concepts ou de méthodologies venus de ces domaines sans s'assurer qu'ils ne représentent pas d'obstacles épistémologiques pour un didacticien, c'est ce que le positionnement impose au chercheur en l'amenant à expliciter les fondements de sa recherche. Les obstacles sont d'ailleurs souvent là où les termes vont de soi!

31 Il nous semble qu'on ne peut pas faire l'économie d'une réflexion sur trois points :

- la conception de la langue ;

- le fonctionnement langagier ;

- le couple apprentissage / acquisition, qui conduit à une réflexion sur la transmission et/ou la médiation.

On constate qu'on retrouve les distinctions de Claude Germain (1993) dans son ouvrage sur l'évolution de l'enseignement des langues : conception de la langue, conception de l'apprentissage, conception de l'enseignement, conception de la relation pédagogique.

33 Nous expliquons la diversité des positionnements par le fait que les trois points mentionnés ci-avant sont décrits de façons très différentes dans les domaines auxquels on peut se référer. Les conceptions dont parle Germain devraient donc toujours être explicitées clairement avec les références voulues. Peut-on négliger les apports de la neuro ou de la psycholinguistique par exemple, mais comment les prendre en compte? Voilà des questions qui méritent réflexion, parmi d'autres.

Les divers axes que Seliger et Shohamy (1989) ont proposés et qui ont été repris et modifiés dans Narcy-Combes (2005: 99 et svtes) facilitent le positionnement que nous suggérons et soulignent son opportunité. En ce qui concerne le positionnement ontologique, le fait d'être relativiste, réaliste non figuratif ou réaliste modifiera profondément la nature des analyses et des conclusions du chercheur et la légitimité qu'il leur attribuera. Un positionnement positiviste rétrécit les choix méthodologiques, pourtant ce positionnement reste encore prégnant dans le monde de la recherche et plus 
encore dans la société au sens large. On ne choisira pas le même type de méthodologie si l'on est déterministe ou volontariste, et on n'attribuera pas la même place aux acteurs. Sur le plan méthodologique lui-même, la complémentarité des méthodologies peut paraitre un enrichissement qui permet de prendre en compte ce qui relève de la nomothétie et ce qui relève de l'idiographie et d'éviter tout dogmatisme théorique, mais il n'y a pas non plus de consensus clair sur ce point, tant dans le monde de la recherche que dans la société en général.

Puisque les chercheurs ont des positionnements différents, qui les conduisent à des problématiques parfois assez éloignées (voir Seidlhofer, 2003), c'est le débat scientifique (publications, colloques) mené selon des critères communs qui permet un dialogue, une comparaison des résultats et des apports et amène chacun à modifier son positionnement et ses concepts de référence si cela lui parait convaincant. Rappelons que le premier de ces critères est que le chercheur en didactique doit préciser ses positionnements en les justifiant / expliquant, en particulier pour ce qui est de la prise en compte des trois contextes suivants :

- contexte personnel / idéologie ;

- contexte de recherche ;

- contexte d'intervention.

Nous ne poserons pas ici plus avant la question de savoir si la didactique est une science, un domaine ou relève d'une autre appellation. Dans la mesure où nous évacuons la reconnaissance institutionnelle de notre réflexion, cela n'a pas d'importance et relève plutôt de la sociologie de la science.

Ayant évoqué les problèmes liés au positionnement épistémologique, nous examinerons maintenant les méthodologies de la didactique des langues.

\section{Méthodologies - Recherche et problématique}

Toute recherche impose au chercheur de construire sa problématique pour se choisir une méthodologie appropriée. Entrent ici en jeu les domaines de référence de la recherche, la triangulation, la question de la validation. Après un examen de ces éléments, nous passerons en revue quelques méthodologies qui nous semblent représentatives et qui correspondent aux orientations de la journée dans laquelle s'inscrit notre contribution.

\subsection{Les domaines de référence}

Le manifeste de l'Acedle parle de "contributions scientifiques de référence". Certains parlent de domaines ou de sciences contributoires. Pothier $(2003$ : 37) présente "la marguerite de la didactique" dans laquelle elle représente "le champ de la didactique et ses rapports avec les disciplines d'intervention". Sans vouloir à tout prix prendre parti, nous avons préféré parler de domaines de référence. L'important pour nous est de ne pas positionner la didactique dans une sorte d'exterritorialité scientifique ou de subordination par rapport aux champs dont elle est proche. En ce sens, nous ne nous reconnaissons pas vraiment dans la " transposition didactique" de Chevallard (1991), pourtant si souvent citée. Un autre terme vient ici à l'esprit: "éclectisme". Nous rejoindrons Denis Lehmann (1997) dans son exigence de cohérence scientifique. 
Persistant ainsi à revendiquer comme nécessaire un soupçon de rationalité dans les relations entre pratiques d'enseignement et recours aux disciplines de référence, j'aurais bien du mal à dissimuler mon peu de goût pour un éclectisme méthodologique aujourd'hui triomphant qui n'est, à tout prendre, que le fruit tardivement mûri, dans notre domaine, de la tentation post-moderne. méthodologie choisie. Rappelons qu'ils relèvent le plus souvent des sciences du langage, des sciences de l'éducation, de la psychologie (clinique ou expérimentale), des sciences sociales, des sciences de l'information et de la communication, de l'informatique (recherches sur les TIC dans leur aspect EIAH, environnements humains d'apprentissage humain).

41 Le concept de recherche scientifique impose qu'on établisse un lien entre les termes d'une théorie et ce qui se passe lors d'une action sur le terrain. Ce travail d'objectivation, passage de l'objet réel, ou sensible (l'action et ce qu'elle implique, tels qu'on se les représente), à l'objet construit (Bachelard, 1936), conduit à prendre ses distances par rapport au langage et aux pensées "de tous les jours"

\subsection{La triangulation}

Certains considèrent que, comme d'autres sciences humaines ou sociales, la didactique doit rechercher une triangulation de ses observations, de ses données ou de ses méthodologies (Juan, 1999), de manière à croiser les approches, à enrichir ses données et augmenter la cohérence de la recherche. Il pourra s'agir, par exemple, d'une triangulation entre quantitatif, qualitatif et observation. Mais il n'y a pas nécessairement accord sur ce point et la triangulation peut aussi être vue comme une forme de positivisme à laquelle il n'est pas nécessaire de s'astreindre.

\subsection{Validité et validation}

Validation et validité, ces deux aspects nous concernent ici. Une méthodologie adéquate doit permettre d'aboutir à des résultats, des conclusions ou des réponses dont on pourra supposer la validité, d'une part en raison de l'adéquation même de la méthodologie retenue et, d'autre part, de par l'adéquation de la conduite de la recherche. On aboutit alors, dans l'idéal, à une validation de la recherche. Cette validation est interne à la communauté des chercheurs (publications, communications...). Une discipline comme la didactique ne recherche-t-elle pas également une validation sociale? On se situe alors non plus au niveau de la recherche seule mais des conséquences de la recherche, de ses effets sur le terrain. Le lien est évident dans une recherche-action, il sera moins clair pour une recherche moins liée à une intervention pédagogique. On peut suivre Develay (2001), entre autres, et penser que la validation sociale est le meilleur garant de l'action et donc de la recherche qui l'a nourrie. Néanmoins, de nombreux facteurs peuvent parasiter une telle validation si elle reste proche d'une mesure de la satisfaction des usagers. Channouf (2004) nous rappelle la difficulté qu'il y a à s'appuyer sur les représentations des individus, et donc sur leur satisfaction, pour évaluer une action, dans la mesure où des influences inconscientes altèrent la perception et l'analyse de ce qui a été vécu. En didactique de L2, on pourrait considérer qu'une recherche est validée socialement, "sur le terrain", si l'on a pu mesurer, dans les termes d'une théorie d'acquisition de L2, une progression des apprenants (cf. Chapelle, 2003, ou Jordan, 2004), et leur satisfaction. On 
pourra aussi, de manière plus souple, considérer, par exemple, qu'un fort degré de participation des apprenants aux activités constitue une validation (au moins dans une première étape, par exemple pour une innovation incluant un travail à distance).

\subsection{La / les méthodologie(s) en didactique des langues}

Dans la variété des recherches, essayons de relever quelques catégories.

\subsubsection{Recherche épistémologique}

Cette recherche est une forme de pré-théorisation. Elle s'interroge sur la manière dont la connaissance se construit dans le domaine, dont les didacticiens construisent leurs programmes de recherche. Elle a été évoquée ci-dessus avec l'ouvrage de Jordan (2004). Le présent texte souhaite se ranger dans cette catégorie, de même que l'ouvrage récent d'un de ses auteurs (Narcy-Combes, 2005).

La recherche épistémologique en didactique des langues doit permettre de mieux gérer les liens entre les domaines de référence en particulier. Le didacticien des langues au départ spécialiste de sciences du langage ou d'acquisition, par exemple, peut avoir tendance à recueillir et traiter des données à partir d'évidences qui s'imposent à lui au sens où Bachelard (1936) le souligne. Il ne prend pas toujours le recul nécessaire pour voir, par exemple, qu'il ne peut traiter certaines données que par une approche qualitative et non quantitative car il ne dispose pas d'un nombre suffisant de sujets. Une telle méconnaissance des méthodologies des sciences sociales est courante de par l'absence de formation dans ces champs dans les cursus suivis par les futurs didacticiens. Une recherche épistémologique doit aussi permettre de rendre le dialogue plus aisé entre divers volets de la recherche didactique ou entre didactique et autres sciences humaines. Si chacun a une vue plus claire de son positionnement, de ses points de départ et de ses choix méthodologiques, ce qui pouvait à première vue sembler des antagonismes ou des incompatibilités se place plus aisément dans une complémentarité où chacun peut s'enrichir des travaux de l'autre. Il s'agit, au sein de la discipline elle-même, de prendre le recul et la distanciation requis pour toute didactique (cf. la citation de Bailly ci-dessus).

47 La recherche épistémologique travaille à partir des publications scientifiques de la didactique et de ses domaines de référence. Cette recherche se traduit par des publications ou communications scientifiques.

\subsubsection{Recherche de synthèse}

Cette recherche propose un état des lieux sur un point ou un concept du domaine. Il peut être difficile de la distinguer de la recherche épistémologique si elle fait le point sur un positionnement théorique ou dans le cas des chercheurs travaillant sur l'histoire de l'enseignement des langues, par exemple. Nous rangerions dans les recherches de synthèse ce qui est suggéré par les thématiques abordées dans les journées "Notions en questions" qui ont précédé celle-ci. La notion de progression, de ressource, l'interactivité, autant de thèmes requérant une synthèse. On pensera aussi aux numéros thématiques de diverses revues. La difficulté, une fois une thématique retenue, est que l'on regroupe diverses contributions et que la synthèse est, peut-être, ce qui va manquer justement si l'auteur de la présentation du numéro ne l'a pas prise en charge pour mettre en perspective les contributions les unes par rapport aux autres. Dans les domaines 
fortement influencés par les effets de mode, les TIC en particulier (notre biais est clair...), on constate un mouvement terminologique et (éventuellement) conceptuel rapide qui nous semble requérir une synthèse justement afin que puissent être situés différents protocoles de recherche ou différents modes d'action les uns par rapport aux autres. On pensera à "autoformation", "hypertexte" et "navigation", "virtuel", "ouvert", par exemple.

La recherche de synthèse est, par ailleurs, celle que doivent nécessairement entreprendre ou ébaucher les enseignants-chercheurs qui doivent assurer des enseignements de didactique. Cette recherche a des points de départ qui sont proches de ceux de la recherche épistémologique. Toutefois on notera qu'ici il ne s'agit plus de s'appuyer uniquement sur des textes scientifiques. Certaines formes de discours "commun", liées à des pratiques, à des instructions officielles deviennent l'un des points d'appui de l'analyse.

\subsubsection{Recherche (quasi) expérimentale}

La didactique des langues se choisit ici une méthodologie qui la rapproche des sciences de l'éducation ou de la psychologie expérimentale, par exemple. L'objectif est de mettre à l'épreuve de l'expérimentation une hypothèse qui sera validée ou non. Cette hypothèse devra être située dans un cadre théorique précisé (nécessité constante du positionnement épistémologique du chercheur). Des données seront recueillies suivant un protocole lui aussi explicité en référence aux cadres théoriques retenus. Ces données devront être aussi contrôlées et prévisibles que possible. Le chercheur devra disposer d'un terrain qu'il peut suffisamment contrôler pour assurer la validité de son travail s'il ne peut travailler en laboratoire (ce qui sera rarement le cas en didactique).

La méthodologie expérimentale fait parfois figure d'idéal inatteignable pour le didacticien ou plus encore pour le praticien chercheur, tant sont nombreux les paramètres qui entrent en jeu pour un apprentissage en milieu institutionnel. Elle semble "véritablement" scientifique à certains en raison de son appui sur des données chiffrées, qui lui donnent une aura indiscutable dans certains cercles. On n'oubliera pas que tout travail quantitatif sera vain s'il n'a pas pris soin de suivre une méthodologie expérimentale adéquate.

Le résultat obtenu ici est la validation ou la non-validation de l'hypothèse dans les conditions expérimentales qui ont été créées. À partir de là le chercheur peut tirer diverses conclusions qui permettront de nourrir d'autres expérimentations ou d'autres types de recherches selon le type d'analyse que l'on fera et les conséquences que l'on souhaitera tirer.

\subsubsection{Recherche-action}

La recherche-action se positionne souvent en contraste à la recherche expérimentale, il serait plus juste, selon Juan, de dire qu'elle relève de l'expérimentation, mais d'un point de vue compréhensif (Juan 1999 : 13). Son approche est qualitative plus que quantitative. Le chercheur est partie prenante de l'action et non "simple" observateur extérieur. Il ne s'agit pas toujours de valider une hypothèse mais plutôt de tenter de répondre à certaines questions de recherche liées étroitement à une pratique pédagogique. Le praticien chercheur de la recherche-action met en place un certain type d'environnement d'apprentissage ou de tâches ou activités. Il définit précisément en quoi et pourquoi ses 
choix devraient avoir un effet positif. Le positionnement épistémologique et le recours à une problématique de recherche restent impératifs si l'on se place dans le cadre de la recherche-action telle qu'elle est pratiquée en France, de manière à entrer dans les cadres institutionnels et conceptuels de la recherche nationale. Ici les points de départ et les données sont évidemment plus nombreux et surtout plus divers, foisonnants parfois, moins contrôlables que dans une recherche expérimentale. Le chercheur acteur doit faire face aux aléas du terrain, s'adapter et modifier éventuellement certaines de ses orientations. La publication de la recherche est ici encore impérative car seule la mise en forme et la structuration requises par l'écrit permettront de mener à son terme la réflexion engagée.

\subsubsection{Recherche développement}

La recherche développement dans son sens de départ a pour objectif de créer un objet qui pourra ensuite être utilisé par d'autres que son ou ses créateurs, auprès d'utilisateurs (apprenants et enseignants donc pour la didactique). On passera par des phases de prototype puis d'expérimentation, de validation et de diffusion. Dans de nombreux domaines la recherche développement donne lieu à des dépôts de brevets, à des collaborations université entreprise, à des commercialisations. On en est, le plus souvent, loin en didactique des langues. Les didacticiens semblent bien absents du développement de matériel pédagogique. Lorsqu'ils sont associés à des projets on les laisse rarement opérer comme chercheurs, avec ce que cela implique pour agir en tant que tels. Ils sont souvent de simples "prête-noms" donnant un vernis d'image universitaire à un produit qui n'est pas développé à l'intérieur de l'université comme projet de recherche. Lorsque le développement se fait dans le cadre d'une recherche, on le voit difficilement déboucher sur une diffusion large, le produit étant par trop artisanal et les collaborations nécessaires ne s'ébauchant pas.

\section{Données et terrain}

La didactique est liée à un terrain (idiographie), ou au terrain (nomothétie), que l'on appelle souvent contexte. Le didacticien tire les données sur lesquelles il travaille de ce terrain. Les recherches épistémologiques et de synthèse signalées ci-dessus sont un cas particulier, mais elles exigent néanmoins de garder en mémoire qu'un terrain existe, et que l'on a affaire à une praxéologie. Le chercheur opère soit directement dans ce terrain, soit indirectement. Nous avons vu qu'en raison de la nature de son objet, et de sa position face à cet objet, sa façon de le voir est modifiée. Le chercheur doit trouver un moyen de traiter les données qu'il recueille de manière adéquate (suivant la méthodologie qu'il aura retenue) en fonction de son objectif, de ses hypothèses ou de ses questions de recherche. S'il rationalise, il trouvera un enchaînement logique qu'il justifiera dans une relation de parfaite causalité. Or, nous avons vu que le jeu entre engagement et distanciation ne relève pas de ce type de raisonnement. Le chercheur n'est sans doute pas dans tel terrain, face à telles données, par hasard. Pour des raisons liées, en partie au moins, à sa curiosité et ses intérêts, il souhaite travailler sur ces données et dans ce terrain. Il y a souvent inévitablement, là, une source de tension dont il faudrait pouvoir mesurer les effets. Cette tension explique la manière dont se construisent le positionnement et le choix qui est fait entre les méthodologies (expérimentale, recherche-action, recherche et développement, synthèse, etc.). Néanmoins, si l'on sort du cadre d'une méthodologie reconnue par sa 
communauté d'appartenance, on s'exclut de cette communauté. Or, il est difficile de ne pas le répéter, dans le cas des sciences humaines, on est loin d'œuvrer en période de "science normale", ce qui peut parfois conduire à des querelles entres les tenants de diverses méthodologies (Mucchielli, 2002, ou Seidlhofer, 2003). Les "intérêts" d'un chercheur risquent d'entrer en conflit avec ce que son positionnement le mènerait à faire parce qu'il souhaite légitimer socialement sa recherche selon des critères qui n'étaient pas les siens initialement. Le souci, ou le besoin, d'être reconnu par la communauté d'appartenance, les comptes que l'on a à rendre aux organismes financeurs ou au terrain, peuvent imposer la nécessité de valider la recherche, en triangulant les analyses de données, en particulier, pour être plus convaincant, ou parce que le terrain ne permet pas de rester dans un cadre unique. On peut se demander s'il est possible d'être totalement quantitatif en quasi-expérimentation ou totalement qualitatif en recherche-action. $\mathrm{Ce}$ souci, ou besoin, conduit également à remettre régulièrement son positionnement en cause, et donc à changer de méthodologie.

\section{Conclusion}

En conclusion, nous pensons qu'autant le positionnement relève du chercheur et de son parcours, autant, la méthodologie qu'il suit relève d'un dialogue qu'il entend établir avec une communauté de chercheurs et avec les terrains sociaux dans lesquels, ou pour lesquels, il œuvre. La cohérence de ses choix méthodologiques est donc impérative puisqu'en un sens elle est le garant de la rationalité de son entreprise. L'humain n'est pas totalement rationnel, les groupes sociaux non plus, on parle de rationalité limitée en économie. On aimerait penser que c'est par leur respect justifié et validé d'une méthodologie reconnue, et choisie parmi d'autres, que les chercheurs peuvent compenser cet état de fait et mieux comprendre et gérer les phénomènes. Maintenir une rigueur épistémologique et méthodologique légitimée par des échanges scientifiques ouverts aux débats devrait, avec le temps, permettre aux didacticiens de faire mieux entendre leur voix à une époque où les décisions politiques se font trop souvent sans prendre en compte les résultats des recherches du domaine ou en les appliquant de façon qui peut les dénaturer (langues dans le primaire, certification, etc.).

\section{BIBLIOGRAPHIE}

Acedle (nd). La recherche en didactique des langues en France - Manifeste pour le XXI ${ }^{\mathrm{e}}$ siècle. Accessible sur le site de l'Acedle : http://acedle.u-strasbg.fr/article.php3?id_article=163 (lien valide à la date de publication).

Bachelard, G. (1938). La formation de l'esprit scientifique. Paris : J. Vrin.

Bailly, D. (1997). Didactique de l'anglais (1) - Objectifs et contenus de l'enseignement. Paris : Nathan.

Callon, M. \& Latour, B. (1991). La science telle qu'elle se fait. Paris : La découverte. 
Channouf, A. (2004). Les influences inconscientes. Paris : Armand Colin.

Chevallard, Y. (1991). La transposition didactique - Du savoir savant au savoir enseigné. Paris : La pensée sauvage.

Depover, C., Giardina, M. \& Marton, P. (1998). Les environnements d'apprentissage multimédia Analyse et conception. Paris : L'Harmattan.

Descartes, R. (1637). Discours de la méthode. (réédition 1966) Paris : Garnier Flammarion.

Develay, M. (2001). Propos sur les sciences de l'éducation. Issy-les-Moulineaux : ESF.

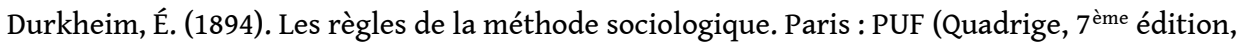
1981).

Germain, C. (1993). Évolution de l'enseignement des langues - 5000 ans d'histoire. Paris : CLÉ International.

Jordan, G. (2004). Theory Construction in Second Language Acquisition. Amsterdam : John Benjamins.

Juan, S. (1999). Méthodes de recherche en sciences sociohumaines. Paris : PUF.

Kuhn, T. (1970). The Structure of Scientific Revolutions. Chicago : The University of Chicago Press.

Lacroix, B. (2002). "La contribution de Norbert Elias à la construction à l'analyse de la construction sociale. De l'état parlementaire". Polis, vol. 9, pp. 1-20.

LeDoux, J. (2003). Neurobiologie de la personnalité. Paris : Odile Jacob.

Lehmann, D. (1997). "Analyse linguistique et problématique didactique en Français sur Objectifs Spécifiques". Relations théorie / pratique dans la formation et la recherche en didactique des langues étrangères - Les langues vivantes dans le secteur professionnel - Actes de l'Acedle. pp. 143-155.

Mucchielli, L. (2000). "Les sciences humaines sont-elles des sciences (comme les autres ?) - Des communautés scientifiques en débat". Axiales - Regards sur les Sciences de l'Homme, 3, pp. 13-28.

Narcy-Combes, J.-P. (2005). Didactique des langues et TIC - Pour une recherche-action responsable. Paris : Ophrys.

Pothier, M. (2003). Multimédias, dispositifs d'apprentissage et acquisition des langues. Paris : Ophrys.

Puren, C. (1988). Histoire des méthodologies de l'enseignement des langues. Paris : Nathan - CLE.

Puren, C. (1994). La didactique des langues à la croisée des méthodes - Essai sur l'éclectisme.

Paris : Didier.

Seidlhofer, B. (2003). Controversies in Applied Linguistics. Oxford : OUP.

Springer, C. (2004). Plurilinguisme et compétences : décrire, entraîner et certifier. Note de synthèse, HDR. Université Marc Bloch Strasbourg 2.

Seliger, H. W. \& Shohamy, L. (1989). Second Language Research Methods. Oxford : OUP.

Soulet, M.-H. (2003). "Penser l'action en contexte d'incertitude : une alternative à la théorisation des pratiques professionnelles ?". Nouvelles pratiques sociales - Une pragmatique de la théorie, vol. 16, $\mathrm{n}^{\circ}$ 2, pp. 125-141. Disponible en ligne. http://www.erudit.org/revue/nps/2003/v16/n2 (lien valide au 14/01/2019) 


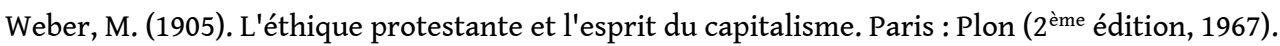

\section{NOTES}

1. Notre regard est ici un regard d'anglicistes.

\section{RÉSUMÉS}

Ce texte, qui introduit une journée d'étude sur le concept de méthodologie de recherche, propose une réflexion épistémologique sur la didactique des langues. Contrastant d'abord méthodologie et méthode, approche compréhensive et explicative, il insiste sur l'importance du positionnement épistémologique du chercheur et souligne que ce positionnement ne peut être indépendant de l'environnement social et de ses pressions. La didactique des langues est située au sein des sciences humaines et sociales et la question de la distanciation et de l'objectivité est analysée. Une complémentarité des approches est suggérée pour dépasser certaines oppositions (idiographie ou nomothétie, quantitatif ou qualitatif). Pour construire sa problématique et choisir une méthodologie appropriée, le chercheur doit s'interroger sur les domaines de référence, la triangulation, la validité. Plusieurs méthodologies sont évoquées dans leur lien aux données et $\mathrm{au}(\mathrm{x})$ terrain(s). La spécificité du parcours de chaque chercheur a un poids important mais la cohérence des choix, la rigueur épistémologique et l'ouverture au débat permettent de participer aux échanges scientifiques.

As an introduction to an academic seminar whose theme is research methodology, this paper presents an epistemological reflection on research in the field of second language learning and teaching. Firstly, the oppositions between methodology and method as well as between comprehensive and explanatory approaches to research are discussed, then, the paper stresses the need for the researcher to make his epistemological position explicit and underlines the fact that this position cannot but depend on the researcher's social environment and the interplay of influence that this fosters. Language learning and teaching research belongs to the larger field of the social sciences and the question of distanciation and objectivity is analysed. The use of various complementary approaches to research is advocated in order to go beyond certain continua (such as the nomothetic - idiographic continuum and the opposition of qualitative and quantitative approaches). In order to be able to construct his theoretical framework and to adopt the most appropriate methodology, the researcher needs to clarify the way he sees the various scientific domains he refers to, triangulation and research validity. The link between the context and the data in various research methodologies will then be discussed. The specific background of each researcher's history plays an important part, but the coherence of the researcher's choices, the solidity of his epistemological position and his openness to debate are what make scientific debates possible. These debates play a constituent role in the construction of scientific knowledge. 


\section{INDEX}

Mots-clés : méthodologie, épistémologie, recherche, didactique des langues

Keywords : methodology, epistemology, research, second language learning and teaching research

\section{AUTEURS}

\section{FRANÇOISE DEMAIZIÈRE}

Françoise Demaizière travaille depuis de nombreuses années sur le lien entre linguistique, didactique des langues et utilisation des technologies pour la formation. Elle encadre des recherches dans le domaine "Apprentissage des langues et systèmes d'information et de communication". Elle participe à la vie de l'Acedle (Association des chercheurs et enseignants didacticiens des langues étrangères) depuis sa création et est membre de l'équipe Diltec (Didactique des langues des textes et des cultures) à l'université Paris 3.

Courriel : francoise.demaiziere[at] univ-paris-diderot.fr

Toile : http://didatic.net

Adresse : Université Paris 7, case 7023, 2 place Jussieu, 75005 Paris, France.

\section{JEAN-PAUL NARCY-COMBES}

Jean-Paul Narcy-Combes est originellement didacticien de l'anglais. Il s'intéresse principalement aux problèmes épistémologiques et méthodologiques soulevés par la didactique des langues, mais également aux tâches et aux dispositifs d'apprentissage. Il encadre des recherches dans ces domaines aussi bien en anglais qu'en FLE, et de plus en plus fréquemment sur une approche commune de l'apprentissage de ces langues à partir d'une même L1. Il est également membre du Diltec.

Courriel : jean-paul.narcy-combes[at]wanadoo.fr Adresse : Université Paris 3, UFR DFLE, Sorbonne, 46 rue St Jacques, 75005 Paris, France. 